\title{
Development of a MATLAB Toolbox for 3-PRS Parallel Robot
}

\author{
Guoqiang Chen and Jianli Kang* \\ Henan Polytechnic University, China \\ jz97cgq@sina.com,kangjl@hpu.edu.cn
}

\begin{abstract}
Aiming at one kind of 3-PRS parallel robot, the study develops a toolbox in MATLAB. The toolbox includes functions for forward kinematics, inverse kinematics, velocity kinematics, error analysis, schematic representation, and so on. The architecture of the 3-PRS robot is introduced firstly. The instructions of the functions, developing procedure and main algorithms are presented secondly. The toolbox encapsulates complicated mathematical formulas into the single function and provides standard inputs and outputs, which improves the reliability and makes it easy to use. Finally, an exanple calls the toolbox function, and verifies its correctness, reliability and convenience.
\end{abstract}

Keywords: 3-PRS parallel robot, toolbox, forward kinematics, inverse kinematics

\section{Introduction}

As an important branch of the industrial robot the ballel robot has many advantages, such as high stiffness, high accuracy little cumulate error, large load carrying capacity and compact structure [1-7]. It has gained wide applications in all kinds of fields and attracted plenty of study. The 3-PRS parallel mechanism is very typical in the parallel robot family [4]. There are many complicated mathematical formulas in analysis on degrees of freedom, working envelope, kinematics, speed, acceleration, accuracy, and so on [8-18]. Especially, the trigonometric function computation, nonlinear equations and complex matrix make design and analysis tedious and discouraging [ , 3-5, 7-18]. The engineering software MATLAB has powerful performance in numerical computation, symbolic operation and graphical representation, so it is an eff cient tool for the robot research [19]. The aim of this paper is to present a computer-aided anarysis toolbox developed in MATLAB for one kind of 3-PRS parallel robot, and giverkey-lgorithms.

\section{Architecture of 3-PRS Parallel Robot}

The schematic representation of the 3-PRS parallel robot is shown in Figure 1 [1, 3-5,7-9]. The robot is composed of a moving platform, three limbs, three vertical rails and a fixed base. Three vertical rails vertically link to the base $B_{1} B_{2} B_{3}$. Moreover, $B_{1} B_{2} B_{3}$ form an equilateral trangle that lies on a circle with the radius $R$. The axis of the revolute pair $\mathrm{R}_{i}$ for $i=1,2$ and 3 is perpendicular to the prismatic pair. Each $\operatorname{limb} \mathrm{L}_{i}$ for $i=1,2$ and 3 with the length of $l_{i}$ connects the corresponding rail by a prismatic pair $\mathrm{R}_{i}$. The moving platform and three limbs are connected by three spherical pairs $b_{1}, b_{2}$ and $b_{3}$. Three spherical pairs form an equilateral triangle that lies on a circle with the radius $r$. The cutter with the length of ${ }_{h}$ is placed at the

*Corresponding Author: Jianli Kang is the corresponding author. Email: kangjl@ hpu.edu.cn

This work is supported by Scientific and Technological Project of Henan Province of China with grant No. 132102210430. 
center of the moving platform. The feed of the prismatic pair is given as $H_{i}$. Angles $\varphi_{i}$ for $i=1,2$ and 3 are defined from the vertical rail to its corresponding $\operatorname{limb} \mathrm{L}_{i}$. As shown in Figure 1, a fixed Cartesian reference coordinate system OXYZ is located at the center $\mathrm{O}$ of the base $\mathrm{B}_{1} \mathrm{~B}_{2} \mathrm{~B}_{3} . \mathrm{X}$-axis and $\mathrm{Y}$-axis are in the base plane $\mathrm{B}_{1} \mathrm{~B}_{2} \mathrm{~B}_{3}, \mathrm{X}$-axis points in the direction of $\mathrm{O}$ $\mathrm{B}_{1}$, and Z-axis is normal to the base plane and points upward. A moving coordinate frame ${ }_{\mathrm{r}_{\mathrm{T}}}$ xyz is located at the cutter point $o_{T}$. The xy plane is parallel to the moving platform $b_{1} b_{2} b_{3}, x-$ axis points in the direction of $\mathrm{c}_{1} \mathrm{~b}_{1}$, and $\mathrm{z}$-axis is normal to the moving platform. The position and orientation of the cutter can be described using the coordinates $\left(x_{\text {Tool }}, y_{\text {Tool }}, z_{\text {Tool }}\right)$ of the cutter point and Euler angles $\alpha, \beta$ and $\gamma$ rotating about Z, Y and X axes of the fixed system. The 3PRS parallel robot possesses 3- DOF that are rotation $\alpha$ about the Z-axis and $\beta$ about the $Y$ axis, and a translational motion $z_{\mathrm{T}}$ along the Z-axis [9]. Three parasitic motions jnclude one rotation $\gamma_{\gamma}$, one translational motion $x_{\mathrm{\tau}}$ about the X-axis, and one translational hotion $y_{\mathrm{r}}$ about the Y-axis. The three parasitic motions $\gamma_{\gamma}, x_{\mathrm{T}}$ and $y_{\mathrm{T}}$ can be expressed using the three independent motions $\alpha, \beta$ and $z_{\mathrm{T}}$.

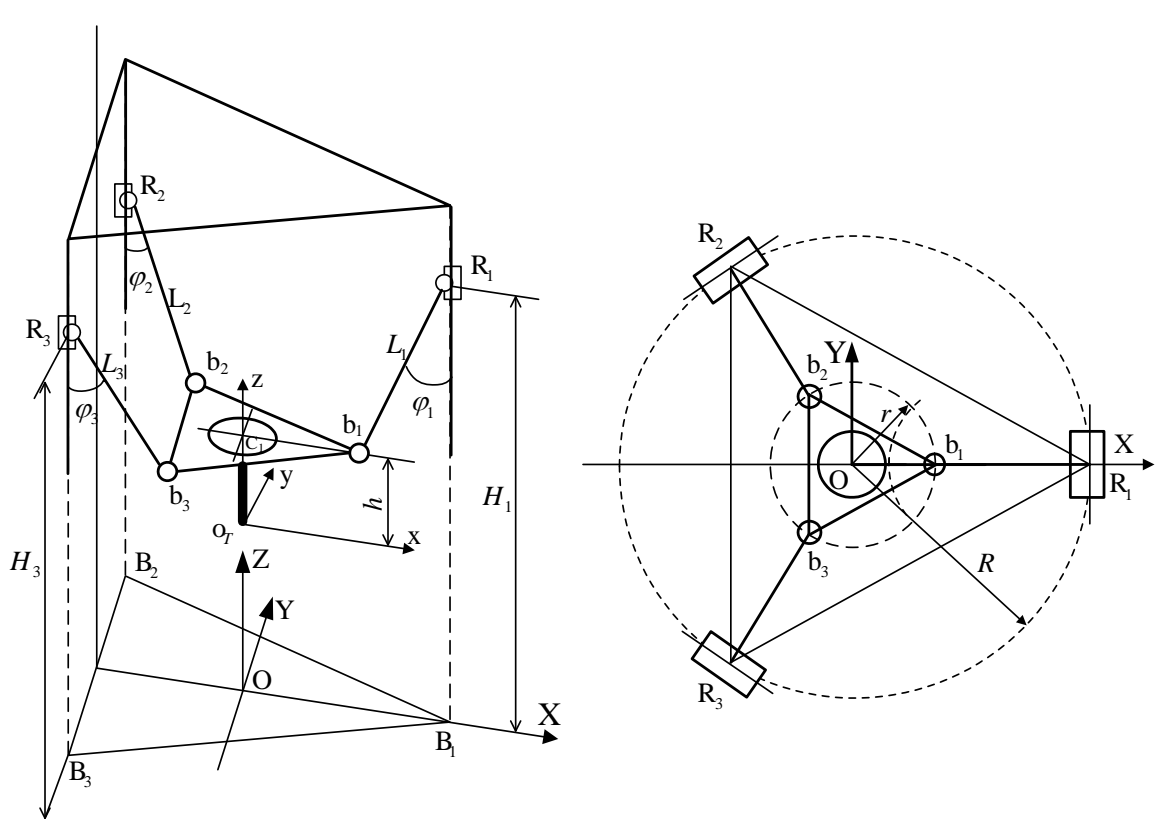

\section{Figure 1. Schematic Representation of the 3-PRS Parallel Robot}

\section{Toolbox Development in MATLAB}

All toolbox function names begin with the prefixion 'TPRS_.' In the toolbox functions, the parameters A, B, G, R, r, h, XT and YT represent $\alpha, \beta, \gamma, R, r, h, x_{\mathrm{T}}$ and $y_{\mathrm{T}}$, respectively. $\mathrm{H}$ is a 1-by-3 vector that represents $\left[H_{1}, H_{2}, H_{3}\right]$. TH is a 1-by-3 vector that represents $\left[\varphi_{1}, \varphi_{2}\right.$, $\left.\varphi_{3}\right]$. B1, B2, B3, R1, R2, R3, b1, b2 and b3 are 1-by-3 vectors of the corresponding points coordinates, respectively. 


\subsection{Function for Homogeneous Transformation Matrix}

$\mathrm{T}=$ TPRS_Tran (A, B, G, P) returns a 3-by-4 array that represents the homogeneous transformation matrix computed using Equation (1) from $\circ_{\tau}$ xyz to OXYZ. The input

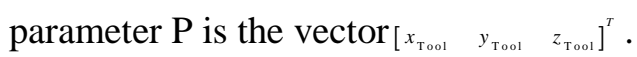

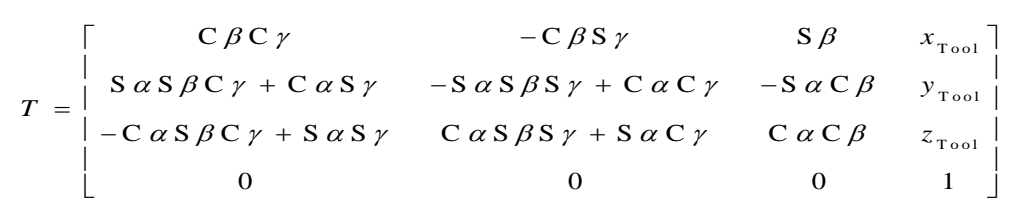

where $\mathrm{s} \alpha, \mathrm{s} \beta, \mathrm{s} \gamma, \mathrm{C} \alpha, \mathrm{C} \beta$ and $\mathrm{c} \gamma$ represent $\sin \alpha, \sin \beta, \sin \gamma, \cos \alpha, \cos \beta$ and $\cos \gamma$ respectively.

\subsection{Function for Euler Angle $\gamma$}

$\mathrm{G}=$ TPRS_AB2G (A, B) returns the Euler angle $\gamma$ that can be expressed by the other two angles $\alpha$ and $\beta$ using Equation (2).

$$
\gamma=-\arctan \frac{\sin \alpha \cdot \min \beta}{\cos \alpha+\cos \beta}
$$

\subsection{Function for Parasitic Motions and $y_{1}$}

$[\mathrm{XT}, \mathrm{YT}]=$ TPRS_XTYT $(\mathrm{A}, \mathrm{B}, \mathrm{C}, \mathrm{h})$ retums the parasitic motions $x_{\mathrm{T}}$ and $y_{\mathrm{T}}$ that can be computed $\beta$ using Equation (3).

$$
\left\{\begin{array}{l}
x_{\mathrm{T}}=\frac{r}{2}(\cos \beta \cos \gamma+\sin \alpha \sin \beta \sin \gamma-\cos \alpha \cos \gamma)-h \sin \beta \\
y_{\mathrm{T}}=h \sin \alpha \cos \beta-r \sin \alpha \sin \beta \cos \gamma-r \cos \alpha \sin \gamma
\end{array}\right.
$$

\subsection{Function for Coordinates of $\mathrm{B} 1, \mathrm{~B} 2$ and $\mathrm{B3}$}

$[\mathrm{B} 1, \mathrm{~B} 2, \mathrm{~B} 3]=\mathrm{TPRS} \mathrm{B} 1 \mathrm{XYZ}(\mathrm{R})$ returns the coordinate vector of B1, B2 and B3 using the following equation system.

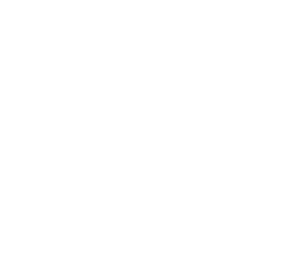

$$
\left\{\begin{array}{l}
\vec{B}_{1}=\left[\begin{array}{lll}
R & O & O
\end{array}\right]^{T} \\
\vec{B}_{2}=\left[\begin{array}{lll}
-\frac{1}{2} R & \frac{\sqrt{3}}{2} R & O
\end{array}\right]^{T} \\
\vec{B}_{3}=\left[\begin{array}{lll}
-\frac{1}{2} R & -\frac{\sqrt{3}}{2} R & O
\end{array}\right]^{T}
\end{array}\right.
$$

\subsection{Function for Coordinates of $R 1, R 2$ and $R 3$}

[R1, R2, R3]= TPRS_RiXYZ (H, R) returns the coordinate vector of R1, R2 and R3 using the following equation. 


$$
\left\{\begin{array}{l}
\vec{R}_{1}=\left[\begin{array}{lll}
R & 0 & H_{1}
\end{array}\right]^{T} \\
\vec{R}_{2}=\left[\begin{array}{lll}
-\frac{1}{2} R & \frac{\sqrt{3}}{2} R & H_{2}
\end{array}\right]^{T} \\
\vec{R}_{3}= \\
{\left[\begin{array}{lll}
-\frac{1}{2} R & -\frac{\sqrt{3}}{2} R & H_{3}
\end{array}\right]^{T}}
\end{array}\right.
$$

\subsection{Function for Coordinates of $b 1, b 2$ and $b 3$}

$[\mathrm{b} 1, \mathrm{~b} 2, \mathrm{~b} 3]=$ TPRS_ForKinbi $(\mathrm{H}, \mathrm{TH}, \mathrm{PofTPRS})$ returns the coordinate vector of $\mathrm{b} 1, \mathrm{~b} 2$ and $\mathrm{b} 3$. PofTPRS is a vector including 4 elements that represent $t_{R}, l_{1}, l_{2}$ and $l_{3}$

The coordinate vector of b1, b2 and b3 in the system $o_{\mathrm{f}} \mathrm{xyz}$ can be computed using Equation (6). Combined with the transformation matrix $\mathrm{T}$, the coordinate vector in the system OXYZ can be gotten.

\subsection{Function for Angles between Vertical Rails and Corresponding Limbs}

$[T H$, Flag] $]$ TPRS_ForKinTH (H, PofTPRS, TH0) computes three feeds and angles using the optimal ieration algotithm based on based on Equation (7). PofTPRS is a 1-by-5 vector that represent $r, R, l_{1}, l_{2}$ and $l_{3}$. The initial iteration value TH0 is a vector including 3 elements, and has heavyeffect on the rationality of the solution.

$$
\left|\mathbf{b}_{1} \mathbf{b}_{2}\right|=\left|\mathbf{b}_{1} \mathbf{b}_{3}\right|=\left|\mathbf{b}_{2} \mathbf{b}_{3}\right|=\sqrt{3} r
$$

\subsection{Function for the Direction Vector of the z-axis}

$\nabla z=$ TPRS_zVectorFromb1b2b3 (b1, b2, b3) returns the direction vector of the z-axis. The direction vector can be computed using the following formula.

$$
\vec{e}_{3}=\frac{\overrightarrow{b_{1} b_{2}} \times \overrightarrow{b_{2} b_{3}}}{\left|\overrightarrow{b_{1} b_{2}} \times \overrightarrow{b_{2} b_{3}}\right|}=\frac{\overrightarrow{b_{2} b_{3}} \times \overrightarrow{b_{3} b_{1}}}{\left|\overrightarrow{b_{2} b_{3}} \times \overrightarrow{b_{3} b_{1}}\right|}=\frac{\overrightarrow{b_{3} b_{1}} \times \overrightarrow{b_{1} b_{2}}}{\left|\overrightarrow{b_{3} b_{1}} \times \overrightarrow{b_{1} b_{2}}\right|}
$$




\subsection{Function for the Coordinates of the Cutter Point}

[XTool, YTool, ZTool]= TPRS_XTYTZTFromb1b2b3(b1,b2,b3,h,dx,dy) returns the cutter point coordinates $\left(x_{\text {Tool }}, y_{\text {Tool }}, z_{\text {Tool }}\right)$. dx and dy are the offsets $\delta x$ and $\delta \mathrm{y}$ along $\mathrm{x}$-axis and $\mathrm{y}$-axis between the center $c_{1}$ of the equilateral triangle and the perpendicular projection point $c_{2}$ of the cutter point in the equilateral triangle plane, shown in Figure 2. The algorithm can be expressed as follows.

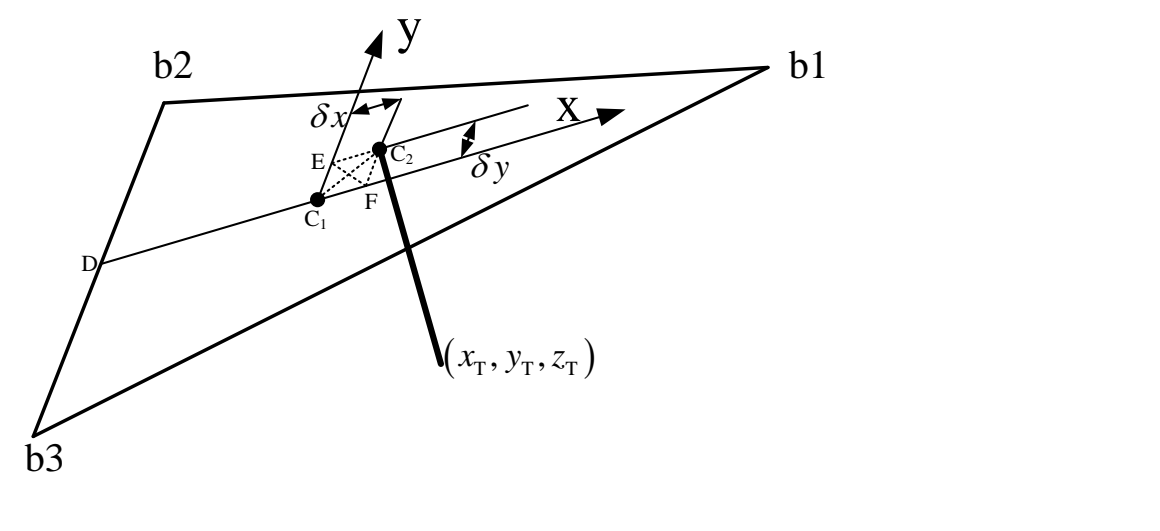

Figure 2. Algorithm Diagram for Function TPRS XTYTZTFromb1b2b3

The coordinates $\left(x_{\mathrm{C} 1}, y_{\mathrm{C} 1}, z_{\mathrm{C} 1}\right)$ of the center $\mathrm{C}_{1}$ of the quilateral triangle b1b2b3 can be expressed as

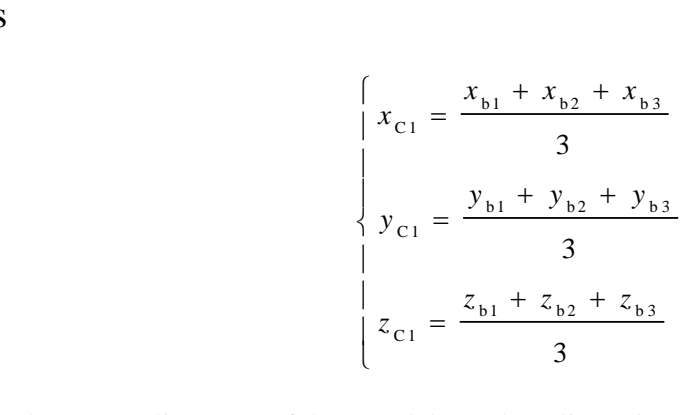

Based on the coordinates of $\mathrm{b} 1$ and $\mathrm{b} 2$, the direction vector $(m, n, p)$ of the $\mathrm{y}$-axis can be computed. The parameter equation for the $y$-axis line is

$$
\left\{\begin{array}{l}
x=x_{\mathrm{C} 1}+m t \\
y=y_{\mathrm{C} 1}+n t \\
z=z_{\mathrm{C} 1}+p t
\end{array}\right.
$$

The corresponding $t$ of the point $\mathrm{E}$ is

$$
t=\frac{\delta y}{\sqrt{m^{2}+n^{2}+p^{2}}}
$$

The coordinates $\left(x_{\mathrm{E}}, y_{\mathrm{E}}, z_{\mathrm{E}}\right)$ of $\mathrm{E}$ can be computed. Likewise, the coordinates of $\mathrm{b} 2 \mathrm{~b} 3$ center $\mathrm{D}$ can be gotten, the direction vector of the $\mathrm{x}$-axis can be computed, and the coordinates 
$\left(x_{\mathrm{F}}, y_{\mathrm{F}}, z_{\mathrm{F}}\right)$ of the point $\mathrm{F}$ can be gotten. Based on coordinates of points $\mathrm{E}$ and $\mathrm{F}$, the center coordinates of the parallelogram $\mathrm{C}_{1} \mathrm{FC}_{2} \mathrm{E}$ can be computed, and coordinates $\left(x_{\mathrm{C} 2}, y_{\mathrm{C} 2}, z_{\mathrm{C} 2}\right)$ of the point $\mathrm{C}_{2}$ are

$$
\left\{\begin{array}{l}
x_{\mathrm{C} 2}=x_{\mathrm{E}}+x_{\mathrm{F}}-x_{\mathrm{C} 1} \\
y_{\mathrm{C} 2}=y_{\mathrm{E}}+y_{\mathrm{F}}-y_{\mathrm{C} 1} \\
z_{\mathrm{C} 2}=z_{\mathrm{E}}+z_{\mathrm{F}}-z_{\mathrm{C} 1}
\end{array}\right.
$$

The function TPRS_zVectorFromb1b2b3 is called to compute the direction vector of the line that the cutter resides. Likewise, $\left(x_{\text {Tool }}, y_{\text {Tool }}, z_{\text {Tool }}\right)$ can be computed.

\subsection{Function for Three Euler Angles from the Direction Vector of the Cutter}

$[\mathrm{A}, \mathrm{B}, \mathrm{G}]=$ TPRS_VectorOfTool2ABG(VectorOfTool) returns Euler angles. The input parameter VectorOfTool is a vector including three elements that represents the direction of the cutter. The 9 upper-left elements in the transform matrix $T$ form the direction cosine matrix from $o_{\mathrm{T}} \mathrm{xyz}$ to OXYZ. The unit vectors of the three coordinate axes of OXYZ are $\vec{E}_{1}=[1,0,0], \vec{E}_{2}=[0,1,0]$ and $\vec{E}_{3}=[0,0,1]$, while the three unit vectors of $\partial_{1} x y z$ are $\vec{e}_{1}, \vec{e}_{2}$ and $\vec{e}_{3}$. The direction cosine matrix $D$ can be expressed as

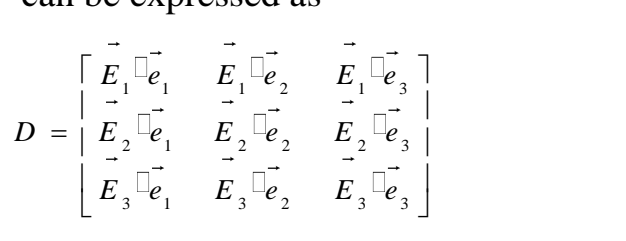

The unit vector $\vec{e}_{3}$ is the utilization one of veetorOfTool. The matrix elements in the third row and third row of ${ }_{D}$ can be computed. The Euler angles $\alpha$ and $\beta$ are computed using Equation (1) and (2), then the Euler angle . Is gotten by calling the function TPRS_AB2G.

\subsection{Function for the Inverse Kinematics}

$[\mathrm{H}, \mathrm{TH}]=$ TPRS_BacKin $(\mathrm{A}, \mathrm{B}, \mathrm{ZT}$, PofTPRS) returns three feeds and three angles. PofTPRS is a vector including 6 elements that represent $r, R, l_{1}, l_{2}, l_{3}$ and $h$. The initial iteration value TH0 is ayeetor including 3 elements, and has heavy effect on the rationality of the solution. The Euler angle $\gamma$ is computed using TPRS_AB2G, and the transformation matrix T is computed using TPRS_Tran. The coordinates of b1, b2 and b3 in the system $o_{\tau_{T}}$ xyz can be expressed as $\left[x_{b i}, y_{b i}, z_{b i}, 1\right]^{T} \quad(i=1,2,3)$, and the corresponding coordinates in the system OXYZ can be computed using the transformation matrix $T$. The feeds of the three prismatic pairs $r(C-1,2,3)$ are

$$
H_{i}=Z_{\mathrm{R} i}=Z_{\mathrm{b} i}+\sqrt{l_{i}^{2}-\left(X_{\mathrm{R} i}-X_{\mathrm{b} i}\right)^{2}-\left(Y_{\mathrm{R} i}-Y_{\mathrm{b} i}\right)^{2}} \quad(i=1,2,3)
$$

where $z_{\mathrm{R} i}$ is the $\mathrm{Z}$ coordinate of $\mathrm{R}_{i}, l_{i}$ is the length of $\operatorname{limb} \mathrm{L}_{i}, X_{\mathrm{R} i}$ and $x_{\mathrm{b} i}$ are the $\mathrm{X}$ coordinates of $\mathrm{R}_{i}$ and $\mathrm{b}_{i}$ respectively, and $Y_{\mathrm{R} i}$ and $Y_{\mathrm{b} i}$ are $\mathrm{Y}$ coordinates of $\mathrm{R}_{i}$ and $\mathrm{b}_{i}$ respectively. 


\subsection{Function for all Solutions of Angles Using Monte Carlo Simulation}

$[\mathrm{TH}, \mathrm{N}]=$ TPRS_ForKinAllTH $(\mathrm{H}$, PofTPRS, THMin, THMax, K, M, Error) returns all solutions of three angles between vertical rails and corresponding limbs using Monte Carlo simulation. The return value $\mathrm{N}$ is the number of the solutions. The input parameter PofTPRS has the same meaning as the function TPRS_BacKin, [THMin, THMax] is the interval where the solution resides, $\mathrm{K}$ is the number of Monte Carlo sampling, and $\mathrm{M}$ is the number of the possible solutions. The solutions are regarded as one if the absolute value of their difference is no more than the limiting error Error. The return number of the solutions $\mathrm{N}$ is no more than the set number $\mathrm{M}$.

\subsection{Function for the Position and Orientation Error}

Error= TPRS_ManError (A, B, ZT, PofTPRS, EPofTPRS) returns the position and orientation error. The return value Error is a vector including 6 elements that represent the errors of the three Euler angles and position coordinates. The input parameter PofTPRS has the same meaning as the function TPRS_BacKin. EPofTPRS is a vector including 11 elements that represent moving platform radius error $\delta r$ fixing base radius error $\delta R$, three limb-length-errors $\delta l_{1}, \delta l_{2}, \delta l_{3}$, cutter length error $\delta h$, threa feeds errors $\delta H f_{3} \delta H_{2}, \delta H_{3}$, offsets $\delta x$ and $\delta y$. The computation procedure is as follows

Step 1: Three feeds $\mathrm{H}$ and three angles TH0 are-computed using [H, TH0]= TPRS_BacKin (A, B, ZT, PofTPRS).

Step 2: The three angles are computed again using [TH, Flag]= TPRS_ForKinTH (H+EPofTPRS(7:9), PofRobot(15)+EPofTPRS (1:5), TH0).

Step 3: The three-dimensional coordinates of b1, b2 and b3 are computed using [b1, b2, b3]= TPRS_ForKinbi(H+EPofTPRS 7:9), TH, PofTPRS(2:5)+ EPofTPRS(2:5)).

Step 4: The direction vector of the $z$-axis is computed using Vz=TPRS_zVectorFromb1b2b3 (b1, b2, b3).

Step 5: Three Euler angles $A, B$ and $G$ are computed using $[A, B, G]=$ TPRS_VectorOfTool2ABG $(-\mathrm{Vz})$.

Step 6: The coordinates $\left(x_{\mathrm{ro}} z_{z_{\mathrm{roo}}}\right)$ are computed using [XTool, YTool, ZTool] = TPRS XTYTZTFromb1b2b3 (b1, b2, b3, EPofTPRS(6), EPofTPRS(10), EPof TPRS(11)).

Step 7: The error Error is computed using the actual and nominal values.

\subsection{Function for the Jacobian Matrix}

Jacobian $=$ TPRS Jacobian $(\mathrm{A}, \mathrm{B}, \mathrm{ZT}$, PofTPRS, Flag) computes the Jacobian matrix according to the position and orientation, and the structure parameter. The input parameter PofTPRS has the same meaning as the function TPRS_BacKin. If Flag is 1, the function returns the Jacobian matrix; else, the inverse Jacobian matrix. The return value Jacobian is a 3 by 3 matrix. The 3-PRS parallel robot possesses 3- DOF $\alpha, \beta$ and $z_{\mathrm{T}}$. Based on the Equation (1) and (14), three feeds are functions of $\alpha, \beta$ and $z_{\mathrm{T}}$. The $\alpha, \beta$ and $z_{\mathrm{T}}$ are functions of the time ${ }_{t}$, and can be expressed as $\alpha(t), \beta(t)$ and $z(t)$. So Equation (14) can be expressed as

$$
H_{i}(t)=F_{i}(\alpha(t), \beta(t), Z(t))
$$




$$
\left\lceil\begin{array}{lll}
\frac{\mathrm{d} H_{1}}{\mathrm{~d} t} & \frac{\mathrm{d} H_{2}}{\mathrm{~d} t} & \frac{\mathrm{d} H_{3}}{\mathrm{~d} t}
\end{array}\right]^{T}=J\left[\begin{array}{lll}
\frac{\mathrm{d} \alpha}{\mathrm{d} t} & \frac{\mathrm{d} \beta}{\mathrm{d} t} & \frac{\mathrm{d} Z}{\mathrm{~d} t}
\end{array}\right]^{T}
$$

where the Jacobian matrix $J$ is

$$
J=\left|\begin{array}{lll}
\frac{\partial H_{1}}{\partial \alpha} & \frac{\partial H_{1}}{\partial \beta} & \frac{\partial H_{1}}{\partial Z}
\end{array}\right|
$$

If $J$ has full rank, the inverse Jacobian matrix can be computed. Because of the complexity of Equations (15) to (17), the Jacobian matrix function jacobian (f, va) be used directly.

\subsection{Function for the Acceleration Computation}

$\mathrm{aH}=$ TPRS_ Acceleration (A, B, ZT,VABX, aABX, PofTPRS) computes the acceleration of three feeds. PofTPRS has the same meaning as the function TPRS_BacKin. VABX and $\mathrm{aABX}$ are the velocity and acceleration vectors of the cutter direction and position, and each includes 3 elements. The return value $\mathrm{aH}$ is a vector including 3 elements representing the acceleration of three feeds. The transmission relationship can be gotten through differentiation using Equation (16).

\subsection{Function for the Structure Diagram Drawing}

TPRS_Plot(B1,B2,B3,R1,R2,R3,b1,b2,b3) plots the scheme of the 3-PRS robot. The function TPRS_XTYTZTRromb1b2b3 iscalled to compute the coordinates of the cutter point, and the MATLAB function plot 3 is used to draw the scheme.

\section{Example Result and Discussion}

The structure parameters of a 3-PRS parallel robot are as follows. $l_{1}=l_{2}=l_{3}=1107, r=200$, $R=350$ and $h=280$. The trajectory path of the cutter point is the intersection of the spherical surface and the plane $z_{0}$, and the cutter is always perpendicular to the spherical surface. The trajectory path of the cutter point can also be expressed as

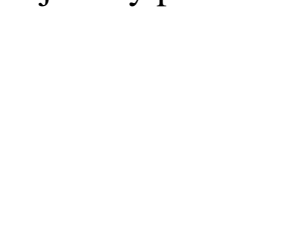

$$
\left\{\begin{array}{l}
x=\sqrt{R_{\mathrm{s}}^{2}-\left(R_{\mathrm{s}}-z_{0}\right)^{2}} \cos t \\
y=\sqrt{R_{\mathrm{s}}^{2}-\left(R_{\mathrm{s}}-z_{0}\right)^{2}} \sin t \quad\left(0^{0} \leq t<360^{\circ}\right) \\
z=z_{0}
\end{array}\right.
$$

The direction vector of the cutter is $-\left(x, y, z_{0}\right)$, and the direction vector of the z-axis is $\left(x, y, z_{0}\right)$ . The toolbox developed can be used to analyze the characteristics. If $R_{s}=100, z_{0}=160$ and $t=60^{\circ}$ , the computation procedure and key codes in MATLAB are as follows. $>$ dRsz0 $=\operatorname{sqrt}(100 * 100-(100-160) *(100-160))$; 


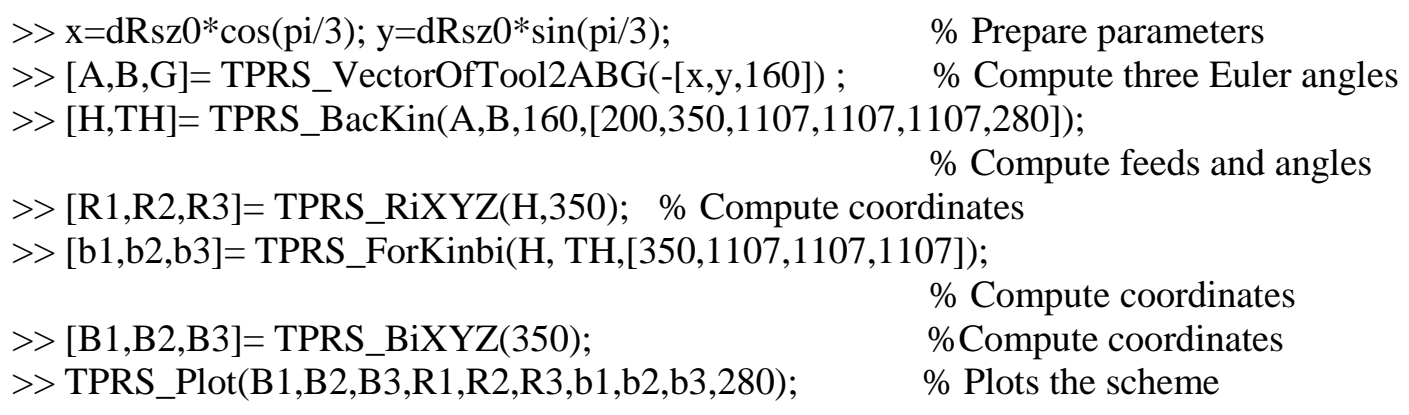

$\%$ Compute coordinates

$\%$ Compute coordinates

The feed vector of the three prismatic pairs is $\mathrm{H}=[1462.50857,1462.50857,1591.87333]$, the three angles vector between vertical rails and corresponding limbs is $\mathrm{TH}=[0.1359$, $0.1359,0.1649$ ], and the scheme of the robot is is shown in Figure 3.

Figure 3. Scheme of the Robot Position and Orientation for Position and Orientation

The paraneter $t$ is made discrete over the interval ${ }_{\left[0^{\circ}, 360^{\circ}\right)}$. Each discretization value is computed, and the result is shown in Figure 3. TPRS_BacKin is used for Figure 4 (a), TPRS_XTYT for Figure 4(b), and TPRS_ManError for Figure 4(c) and (d). The error vector of the 11 parameter is $\varphi .0068,0.0770,0.0798,0.0250,-0.0726,-0.0566,-0.0636,-0.0918$, $0.0788,0.0232,0.0878]$ in Figure 4(c) and (d). The three position-coordinates errors and three Euler angles errors differ greatly in value although the error source is same, which demonstrates that the position and orientation has tremendous effect on the error sensitivity. The translational motion of the 3-PRS robot possesses is only one $z_{\mathrm{T}}$ along the Z-axis, and it has wo parasitic motions $x_{\mathrm{r}}$ and $y_{\mathrm{T}}$, as shown in Figure 4(b). An X-Y table is needed to compensate the parasitic motions $x_{\mathrm{T}}$ and $y_{\mathrm{T}}$, and the compensations Xp and Yp are shown in Figure 4 (b). 


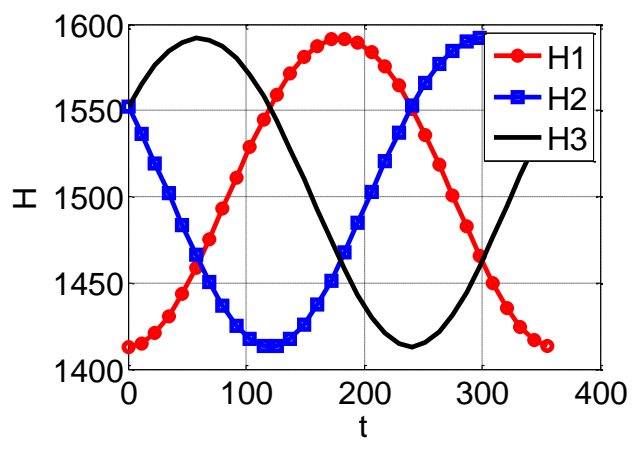

a) Feeds of Three Prismatic Pairs

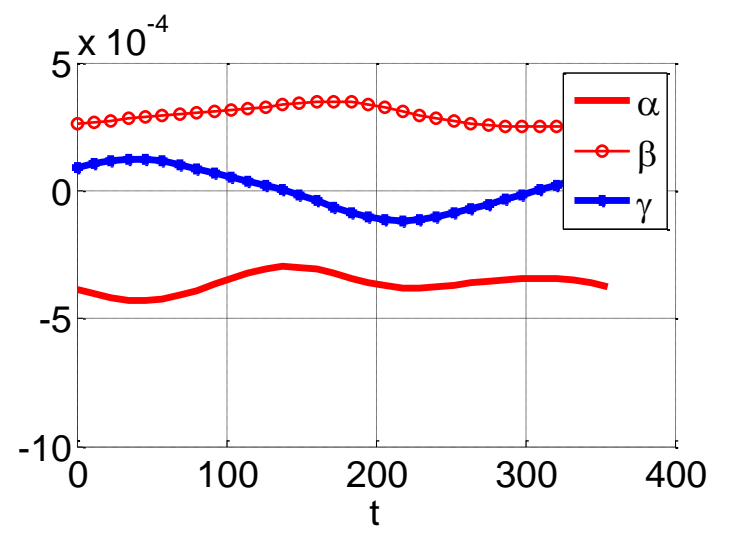

c) Three Euler Angles Errors

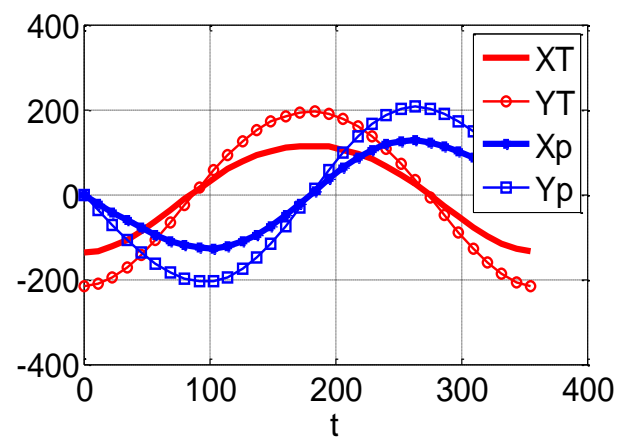

b) Parasitic Motions and Compensations

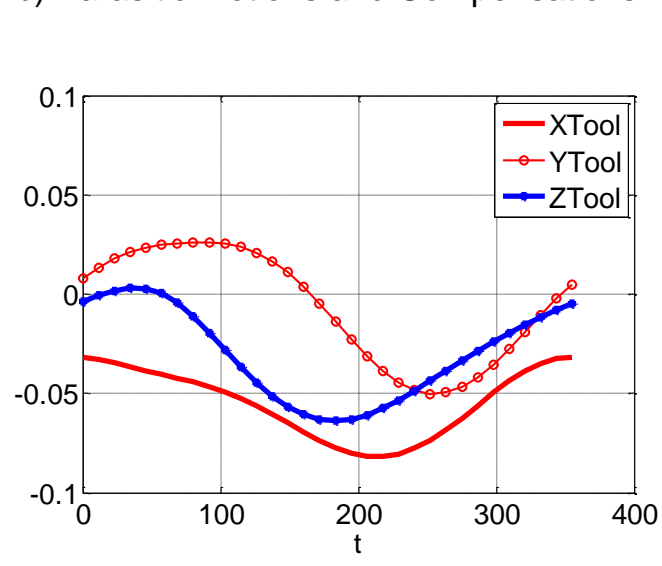

d) Position Errors

Figure 4. Computing Results of the Example

\section{Conclusions}

A toolbox for the 3PRS paralel robot is developed in MATLAB and key algorithms are given. The toolbox includes 16 functions for forward kinematics, inverse kinematics, velocity kinematics, error analysis, Sehematic representation of the robot, and so on. Finally, an example calls the toolbox function, and verifies its correctness, reliability and convenience. The toolbox is very useful for design and analysis of the 3-PRS robot characteristic. The developed toolbox and its application have several advantages. The toolbox encapsulates complicated mathematical formulas into the single function and provides standard inputs and outputs, which improves the reliability and makes it easy to use. The functions are saved as $\mathrm{m}$-files and all file names end with the extension '.m', so it is helpful and almost necessary for the usentomodify the codes for expansion. However, it should be noticed that the 3-PRS robo can be classified into four categories including seven kinds according to limb arrangements as discussed in [9]. Based on the toolbox here, the function for the other kinds can be extended.

\section{Acknowledgements}

This work is supported by Scientific and Technological Project of Henan Province of China with grant No. 132102210430. The authors would like to thank the anonymous reviewers for their valuable work. 


\section{References}

[1] W. Liu and S. Chang, "Study on structural design of a parallel robot and applications in automobiles", Machinery Design \& Manufacture, no. 6, (2012), pp. 141-143.

[2] Z. Huang, "Theory of parallel robot mechanism", 20 Anniversary of Mechanical Science Fundamental Study. Edited by. Y. Lei, Wuhan China: Wuhan University of Technology Press, (2007).

[3] J. Zhao, "Research on the accuracy theory and the measurement device of serial-parallel machine tools", Dotoral dissertation, Wuhan China: Huazhong University of Science and Technology, (2001).

[4] X. Liu, C. Wu , J. Wang and I. BONEV, "Attitude description method of [PP]S type parallel robotic mechanisms", Chinese Journal of Mechanical Engineering, vol. 44, no. 10, (2008), pp. 19-23.

[5] M. S. Tsai and W. H. Yuan, "Dynamic modeling and decentralized control of a 3 PRS parallel mechanism based on constrained robotic analysis", Journal of Intelligent and Robotic Systems: Theory and Applications, vol. 63, no. 3-4, (2011), pp. 525-545.

[6] C. Gong, J. Xiong and C. Huang, "Kinematics and dynamics simulation of Delta parallel robot", Manufacturing Automation, vol. 35, no. 3, (2013), pp. 5-7\&14.

[7] G. Abbasnejad, S. Zarkandi and M. Imani, "Forward kinematics analysis of a 3-PRS parallel manipulator", World Academy of Science, Engineering and Technology, vol. 37, (2010) January, pp. 329-335.

[8] M. S. Tsai, T. N. Shiau, Y. J. Tsai and T. H. Chang, "Direct kinematic analysis of a 3-PRS parallel mechanism”, Mechanism and Machine Theory, vol. 38, no. 1, (2003), pp. 71-83.

[9] Q. Li, Z. Chen, Q. Chen, C. Wu and X. Hu, "Parasitic motion comparison of 3-PRS parallel mechanism with different limb arrangements", Robotics and Computer-Integrated Manufacturing, vol. 27, no. 2, (2011), pp. 389-396.

[10] J. Yu, Y. Hu, S. Bi, G. Zong and W. Zhao, "Kinematics eature analysis of a 3-DOF compliant mechanism for micro manipulation", Chinese Journal of Mechanical Engineering, vol. 17 no. 1, (2004), pp. 127-131.

[11] J. A. Carretero, R. P. Podhorodeski, M. A. Nahon and O. M. Gosselin Kinematic analysis and optimization of a new three degree-of-freedom spatial parallel manipulator", Journa $\varrho$ Mechanical Design, Transactions of the ASME, vol. 122, no. 1, (2000), pp. 17-24.

[12] P. Huang, J. Wang, L. Wang and J. Qian, Dimensional synthesis for 3-PRS mechanism based on identifiability performance", Chinese Journal of Mechanical Engineering, vol. 25, no. 2, (2012), pp. 234-240.

[13] K. H. Hunt, "Structural kinematics of in-parallek-actuated robot-arms", Journal of Mechanisms, Transmissions, and Automation in Design, vol. 105, no. 4, (1983), pp. 705-712.

[14] Y. Li and Q. Xu, "Kinematic analysis of a 3-PRS parallel manipulator", Robotics and Computer-Integrated Manufacturing, vol. 23, no. 4, (2007), pp. 395-408.

[15] D. Liu, R. Che, Z. Li and X. Suo, "Research on the theory and the virtual prototype of 3-DOF parallel-link coordinate-measuring machine', IEEE Transactions on Instrumentation and Measurement, vol. 52, no. 1, (2003), pp. 119-125.

[16] N. A. Pouliot, C. M. Gosselin and M. A. Nahon, "Motion simulation capabilities of three-degree-of-freedom flight simulators", Journal of Aircraft, yol. 35, no. 1, (1998), pp. 9-17.

[17] A. A. Selyakumar, R. P. Babu and R. Sivaramakrishnan, "Simulation and singularity analysis of 3-PRS parallel manipulator". Proceedings of the 9th IEEE International Conference on Mechatronics and Automation, Chengdu Ch na, (2012) August 5-8, pp. 2203-2207.

[18] J. Wahl, 2000. Articulated tool head, US Patent 6,431,802, (2002) August 13.

[19] T. Yu, D. Li and B Song, "Research on trajectory planning and simulation of industrial robots based on MATLAB-Roboties toolbox", Mechanical Engineer, no. 7, (2011), pp. 81-83.

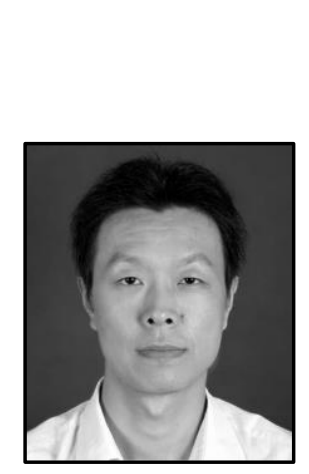

\section{Author}

Guoqiang Chen, is currently an associate professor at School of Mechanical and Power Engineering of Henan Polytechnic University, China. His research interests include robot technology, electric vehicle control, motor control, pulse width modulation technology and software design. 
International Journal of Hybrid Information Technology

Vol.7, No.5 (2014)

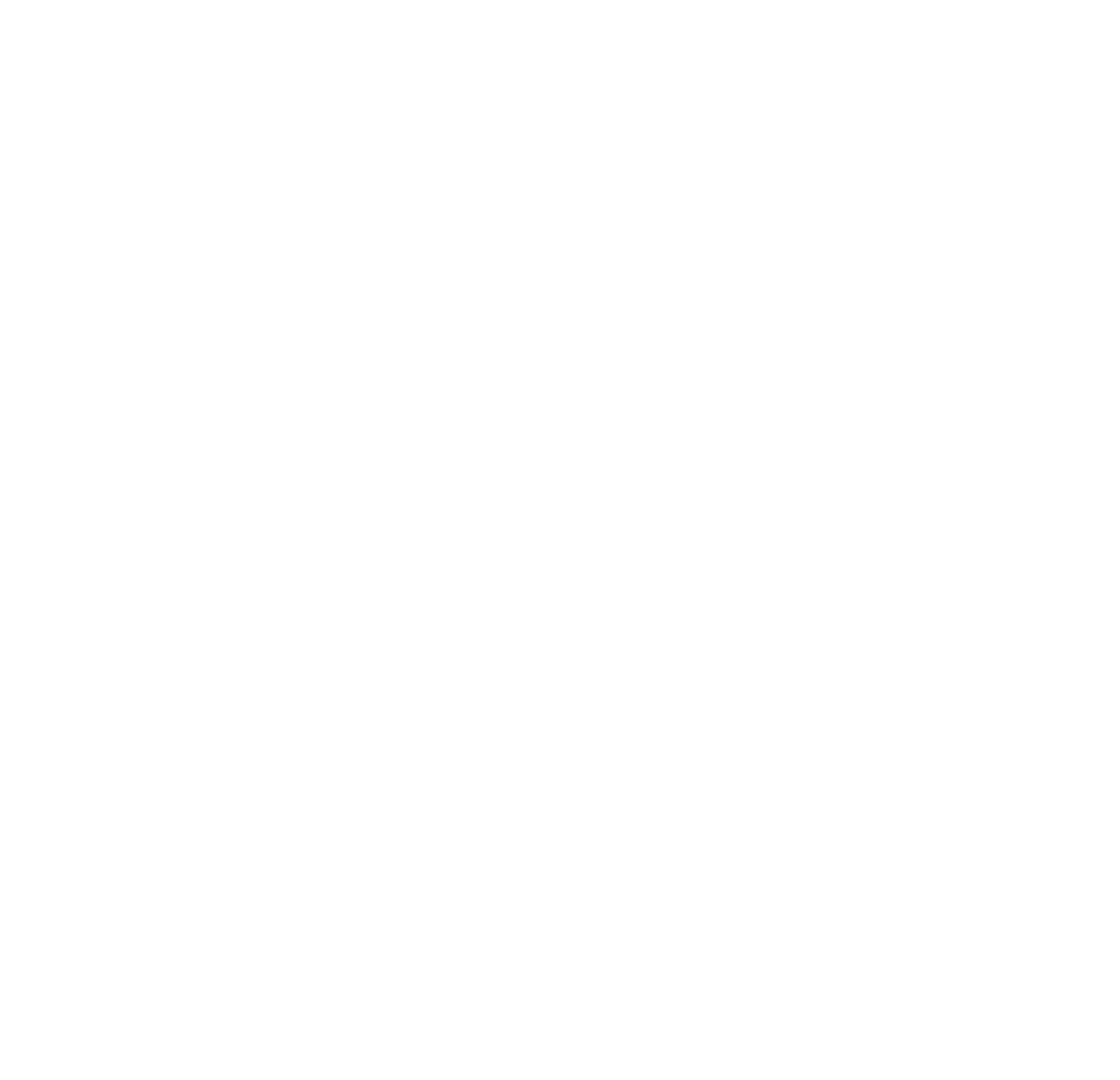

